\title{
Development of a Visual Analytics Tool for Polytrauma Patients: Proof of Concept for a New Assessment Tool Using a Multiple Layer Sankey Diagram in a Single-Center Database
}

\author{
Mica, Ladislav ; Niggli, Cedric ; Bak, Peter ; Yaeli, Avi ; McClain, Margaret ; Lawrie, Charles M ; \\ Pape, Hans-Christoph
}

\begin{abstract}
INTRODUCTION Early physiological assessment of multiple injured patients is crucial for decision making and has relied on personal experience of trauma experts. We have developed a new visual analytics tool (Sankey diagram, Watson Trauma Health care tool) that includes known prognostic parameters for polytrauma patients to help guide assessment and treatment decisions for physicians involved in trauma care. METHODS A prospectively collected trauma database of a single level I trauma center (3655 patients) was used. INCLUSION CRITERIA age $>16$ years, an injury severity score (ISS) $>16$ and presence of a complete data set in the database. Data collected included admission values of patient age, injury scoring, shock classification, temperature, acid-base and hemostasis parameters. All of these parameters were collected daily as longitudinal parameters. Endpoints of the clinical course we considered were sepsis, SIRS and early in hospital mortality $(<72 \mathrm{~h})$. A proof of concept of the visualization was developed over a 2 -year period in a cooperation between physicians and engineers. Statistically, the most predictive parameters were selected by binary logistic regression and ROC analysis. RESULTS A dynamic interactive multilayer Sankey diagram, based on cohort similarities, was developed in a collaboration between the University Hospital of Zurich, Department of Trauma and IBM, from August 2017 until January 2018. It is a modular tool and allows any user to add a new patient, or work with an existing case. The visualization used the data-driven documents (D3) interactive visualization library to create a responsive graphic. CONCLUSIONS This application summarizes the experience of 3655 polytrauma patients and might serve as a guide for clinical decisions and educative purposes, as well as new scientific questions for the polytrauma patient. LEVEL OF EVIDENCE IV.
\end{abstract}

DOI: https://doi.org/10.1007/s00268-019-05267-6

Posted at the Zurich Open Repository and Archive, University of Zurich

ZORA URL: https://doi.org/10.5167/uzh-178500

Journal Article

Accepted Version

Originally published at:

Mica, Ladislav; Niggli, Cedric; Bak, Peter; Yaeli, Avi; McClain, Margaret; Lawrie, Charles M; Pape, HansChristoph (2020). Development of a Visual Analytics Tool for Polytrauma Patients: Proof of Concept for a New Assessment Tool Using a Multiple Layer Sankey Diagram in a Single-Center Database. World Journal of Surgery, 44(3):764-772.

DOI: https://doi.org/10.1007/s00268-019-05267-6 
Development of a visual analytics tool for polytrauma patients -proof of concept for a new assessment tool using a multiple layer Sankey diagram in a single centre database

Ladislav Mica ${ }^{1}$ - Cedric Niggli ${ }^{1}$ Peter $\mathrm{Bak}^{2}$ • Avi Yaeli ${ }^{2}$ Margaret McClain ${ }^{3} \bullet$

Charles M. Lawrie ${ }^{4} \cdot$ Hans-Christoph Pape ${ }^{1}$

${ }^{1}$ Department of Trauma Surgery, University Hospital of Zurich, 8091 Zurich, Switzerland

${ }^{2}$ IBM Haifa University Campus, Mount Carmel Haifa, 3498825, Israel

${ }^{3}$ Gettysburg College, Durham University, 281 Canterwood Ln, Wexford, PA 15090, USA

${ }^{4}$ Dept. of Orthopedic Surgery, Washington University School of Medicine, St. Louis MO 63130, USA

\section{Corresponding author:}

PD Dr.med. Ladislav Mica, PhD, FEBS, FACS

Division of Trauma Surgery

University Hospital Zurich

Ramistrasse 100

8091 Zurich

Switzerland

ladmic@gmail.com

Mob: 0041792103164 


\section{Abstract}

\section{Introduction}

Early physiological assessment of multiply injured patients is crucial for decision-making and has relied on personal experience of trauma experts. We have developed a new visual analytics tool (Sankey diagram, Watson Trauma Health care tool) that includes known prognostic parameters for polytrauma patients to help guide assessment and treatment decisions for physicians involved in trauma care.

\section{Methods}

A prospectively collected trauma database of a single level I trauma center (3655 patients) was used. Inclusion criteria: age > 16 years, an Injury Severity Score (ISS) > 16, and presence of a complete data set in the database. Data collected included admission values of patient age, injury scoring, shock classification, temperature, acid-base and hemostasis parameters. All of these parameters were collected daily as longitudinal parameters. Endpoints of the clinical course we considered were Sepsis, SIRS and early in hospital mortality (< 72 hours). A proof of concept of the visualization was developed over a 2-year period in a cooperation between physicians and engineers. Statistics The most predictive parameters were selected by binary logistic regression and ROC analysis.

\section{Results}

A dynamic interactive multi-layer Sankey diagram, based on cohort similarities, was developed in a collaboration between the University Hospital of Zurich, Dept. of Trauma and IBM, from Aug 2017 until January 2018. It is a modular tool and allows any user to add a new patient, or work with an existing case. The visualisation used the Data-Driven Documents (D3) interactive visualisation library to create a responsive graphic. 


\section{Conclusions}

This application summarizes the experience of 3655 polytrauma patients and might serve as a guide for clinical decisions and educative purposes, as well as new scientific questions for the polytrauma patient.

Level of evidence: IV. 


\section{Introduction}

Large databases have been used successfully to determine risk profiles for trauma patients [13] . The most frequent approach is a deductive association between injury profiles and complication rates, usually performed in a static fashion at the time of admission [4-6]. Use of a dynamic approach appears to be exception to the rule for most studies. In addition, few trauma scoring systems have included physiological parameters [7-9]. The data obtained is often utilized to solve research questions by those involved in trauma care, or to assess patient populations [10-12]. Most frequently, the information is obtained by score value results, i.e., values are expressed as percentages that describe particular risk scenarios [13-15].

For the prediction of outcomes in patients with multiple injuries, special circumstances occur, as multiple independent factors can affect the outcome, such as injuries or injury combinations, rescue conditions, preexisting morbidities, and certain laboratory values on admission $[16,17]$. Notably, a variety of laboratory values have been found to be affected by other measures, influencing the clinical course [18]. The complexity may also increase due to time-dependent changes in parameters, and by those induced by clinical decision making and treatments, such as initiation of major hemorrhage protocols and decisions regarding surgery $[19,20]$

Along with the availability of larger databases, attempts have been made to reduce the complexity by using computerized validation processes, or preexisting databases that provide multiple layers of information [21]. One way of improving understanding while dealing with several levels of information might be to visualize the multiple levels in a Sankey diagram. Historically, the name Sankey derives from an Irish naval officer, who used the visualization tool to investigate the efficiency levels of steam engines [22]. The concept was modified 
through the inclusion of additional time-dependent factors. As a result, a directory tree was added to animate the image through time [23-25]. In clinical medicine, there is a paucity of reports using this strategy. To date, a few attempts have been made to incorporate the Sankey technique into clinical medicine by an IBM computer system, Watson [26]. Watson has been used to describe general health issues [27], to model medical care plans, and to visualize the development of community acquired pneumonia leading to hospitalization [28].

The use of data sets to develop an interactive visualization tool of clinical trauma scenarios has not previously been available. We therefore developed a visual analytics approach based on the IBM Watson Health tool and an existing polytrauma patient database. This approach has undergone multiple steps of development in a close interaction between physicians and engineers. This current study hypothesized that this development might provide proof of concept, facilitating the projection of patient scenarios for severely injured patients admitted to a trauma center. 


\section{Methods}

Prior to the initiation of the study, an independent review regarding clinically relevant parameters revealed injury severity, coagulation, acid-base changes, and shock, e.g., advanced trauma life support (ATLS) scoring was made [21-29] (Table 1). All parameters deemed clinically relevant were included in the Sankey diagram, and these parameters were also were applied for longitudinal scoring options. The visual parameters were integrated by three developers at IBM (CN, PB and AY).

\section{Ethical approval}

Ethical approval was obtained from the local review board at USZ (Nr. St.V. 01-2008). The study was conducted according to the guidelines for good clinical practice, recommended by the Swiss National Ethics Committee.

\section{Sample collection}

Proof of concept was performed on the basis of an institutional database of trauma patients $>16$ years of age, with an ISS $>16$. The database was established on 01.08 .1996 with ongoing patient data collection, as described previously [5]. For this study, inclusion criteria were patients aged 16-75 years, and completeness of all data at admission and longitudinally during the first three weeks after injury (Table 2). Data collected for analysis included laboratory values from admission until 21 days post injury, and included clinical scoring systems as described below.

\section{Definitions and Scoring systems}

The ISS, based on the abbreviated injury scale (AIS; update 2008 version), was used to determine regional injuries, and it was also used for grading general severity of trauma [30]. 
Shock states (I-IV) were defined according to criteria used by ATLS, an established scoring system widely used for medical teaching.

SIRS was defined as the presence of the following criteria: body temperature $>38{ }^{\circ} \mathrm{C}$, heart rate $>90 \mathrm{bpm}$, respiratory rate $>20$ breaths/ min or $\mathrm{PaCO}_{2}<32 \mathrm{mmHg}$, and neutrophil count $>12,000 / \mathrm{ml}$ or $<4,000 / \mathrm{ml}$. Likewise, sepsis was diagnosed by a previous scoring system [31, 32].

End points

Independent predictors for the outcome parameters, SIRS, sepsis and early death, were used.

Time line of development of the "Watson Health Trauma" tool

The ideas to develop a new assessment tool for trauma patients were generated in 2015 , as existing databases provided only limited information about predictive parameters [33, 34]. With the IBM Watson tool [35] and the availability of an existing database, the ideas about the new application developed in 2017. Consequently, an application for funding to enable the implementation of trauma patient parameters into the existing pathway concept was made. The results of further refinements, including input from surgeons and engineers, are summarized in Table 1.

\section{The development of the visual pathway}

Visualization was based on a variation of a Sankey diagram, which allows the user to explore the distribution, sequence and relationship of clinical scenarios of interest for outcomes and other events, as described in the introduction.

Figure 1 provides an overview of the architectural components of the system. In order to deal with the complexity and considerable variance found in the raw data, a data ingestion and transformation engine was developed by engineers. It included the following 
algorithms; medical code grouping, statistical frequency grouping, time interval simplification, regimen extraction, consecutive event merging, and event filtering. Following simplification of the pathways, frequencies and ratios at the level of the cohort were summarized. The data ingestion loaded the data into a client-side internal storage, where the user could filter and visualize it. The client-side had been implemented as a web application using standard JavaScript (JS) components. An angular JS library was used to coordinate the application components and connect to the server side for data requests and querying [36]. A bootstrap cascading style sheet (CSS) and JS were used to achieve interactivity of the application and for styling the application widgets, such as selection and slider components [37]. The application was able to export data streams into MS-Excel documents in a standardized format.

Within the application, any user can add a new patient, or work with an existing case. A case represented a medically relevant description of a patient of interest. The mandatory data included age, body temperature, and ISS. The filter criteria could be extended to lab-test results, such as lactate, pH, hemoglobin, hematocrit, and AIS scores. With every change of the filter criteria, a new query request was sent to the server and the relevant cohort retrieved for exploration, resulting in different insights and cohort views, which were added to the case and stored on the server.

The tool used the Data-Driven Documents (D3) interactive visualization library to create a responsive graphic [38]. The information was organized in a node-link flow diagram, a variant of a Sankey diagram, defined by the trauma surgeon and uniquely designed for this application. Each node in the graph represented a medical state, such as a treatment or outcome, or any other medical state of interest. The links represented transitions between consecutive states in the cohort with a relation to the clinical reality. An example of the visualization and the filter components can be seen in Figure 1. The height of nodes and 
links in the Sankey diagram represented the relative number of patients that shared the medical state and transition, respectively. The color was mapped to the relative proportion of patients that presented with an outcome of interest, such as SIRS (Figure 2.).

\section{Statistics}

All statistical analysis was performed by IBM engineers to justify distribution of patients according to clinical scores (e.g. ATLS classes etc.). In preparation an analysis for normal distributions was performed and the predictive quality of the different variables was tested by receiver operating characteristic ROC curve analysis. The independent predictive power was analysed using binary logistic regression. The goodness of fit for the binary logistic regression was analysed by the Hosmer-Lemeshow test, and was considered to be good if $p>0.05$. Data were analysed using IBM SPSS ${ }^{\circledR}$ Statistics for Windows software version 23.0 (IBM Corp., Armonk, NY, USA). 


\section{Results}

Based on the preparative literature review, the factors with the highest impact on outcomes for patients with multiple injuries were reconfirmed from previous reports: ISS, pH, lactate, haemoglobin, haematocrit, temperature and patient age and were included in the development (Table 2) [1-20, 30-35, 39-44].

The database contains information on 3655 multiply injured patients [5]. Data stratification led to the exclusion of 1730 patients, leaving 1925 patients for assessment in the current study. These data sets were excluded due to data lacking in patient profiles, such as the temperature at admission or incomplete documentation of fluid management.

Primary output and cohort selection for patients with multiple injuries

The primary output of the visual analytics tool was a Sankey diagram. It provided a nontemporal event sequence from the input parameters and groups listed above (see visual pathway analytics, Figure 1), as well as the following categories (parameters): coagulopathy (prothrombin time), haemorrhagic shock (ATLS groups I to IV), surgical decision making (whether damage control, early total care or no surgical intervention) and outcome (SIRS, sepsis and early death; Table 2). 


\section{Discussion}

High energy injuries continue to represent a significant cause of death and long-term disability, even in high-income countries [41]. While prognosis based on sequential laboratory parameters is well known to be relevant, the initial values are the only prognostic tool in the early stages after admission. Several scoring systems have been developed in the past. Our group has recently shown that the use of multiple parameters, covering coagulopathy, indices of shock soft tissue injuries and acid base changes outweigh the use of a single parameter [42].

The clinical coverage of multiple diagnostic tools may be difficult to overlook and while experienced physicians are thought to be able to integrate and manage multiple sets of information, physicians in training may benefit from getting help in overviewing them. In this line, visual analytics might provide a way to facilitate the interface between the user, the analytic system, and the underlying data. The value of visual analytics should be assessed in the context of the domain, available data, and suitability of visualization for the given user tasks.

We propose that the results presented in this manuscript represent a proof of concept in determining a different approach in patient assessment. However, we are aware that certain important limitations apply. First, the inclusion criteria of the study focused on patients between 17 and 74 years of age, thus excluding those outside this age group from using the tool. Second, we had to limit the end points to complications available in the database. As the definitions for some complications, such as acute respiratory distress syndrome (ARDS), have changed during the processing of the data collection [3], they were not included in the outcome assessment. Third and most importantly, inclusion of patients from as long ago as 
1996 might represent a drawback, as management strategies have changed as well. Previous attempts to use more current databases led to the use of a trauma registry, which was limited by the availability of longitudinal data. In addition, other important laboratory parameters, e.g., rotational thromboelastometry (ROTEM) and interleukin values, described in recent reports, were not available in the trauma registry or in the current data set. Therefore, we propose that the current results might be used as a proof of concept that justifies further analysis.

In certain ways, our visualization tool might be an improvement on previous efforts to develop scoring systems for trauma patients. Most of the available trauma scoring systems either rely on a single parameter, or focus on data from the time of admission or after completion of resuscitation. In a previous review of parameters indicative of clinical patient status, we described options to assess patients and listed the available recommendations regarding haemorrhagic shock, coagulopathy, hypothermia and soft tissue injuries [43], similar to those previously pointed out by Border [44]. These recommendations were applied, usually before decision making regarding surgical procedures. Ogura et al. used large databases to describe the degree of hemorrhage, but did not specify the time of application [45]. However, none of these scoring systems, or compilations of data considered the entire hospital stay or the diagnosis of patients.

Our newly developed Sankey visualization tool might provide an improvement over these previously described scoring systems by providing different scenarios, and potentially being a tool when speaking with families regarding decision making. In addition, if validated appropriately in the future, it might be helpful in considering several potential outcomes for any given clinical scenario encountered by the treating physician. 
We propose that our development process, which included multiple adaptations of the visualization and Sankey configuration, have been helpful in improving clarity and ease of use. In the current state of development, it is unable to provide any distinct treatment recommendations that deal with causality, confounders, or statistical/human biases. In addition, it should not be viewed as a medical device from a regulatory perspective. In order to improve the reliability of the selected parameters, we are currently testing for validation and applicability. This approach should also be helpful in improving guidance regarding the clinical course of patients. We anticipate that future validation will further improve the prognostication options.

Conflict of interest The authors declare no conflict of interest related to the submitted study.

Ethical agreement Permission: "Retrospektive Analysen in der Chirurgischen Intensivmedizin” Nr. St.V. 01-2008. Ladislav Mica

Acknowledgements Innovation Funding INOV00040; University Hospital Zurich; 8091 Zurich; Switzerland; 06/2017; Ladislav Mica 


\section{References}

1. Champion HR, Copes WS, Sacco WJ, et al (1990) The Major Trauma Outcome Study: establishing national norms for trauma care. J Trauma 30(11):1356-1365

2. Sauaia A1, Moore EE, Johnson JL, et al (2014) Temporal trends of postinjury multiple-organ failure: still resource intensive, morbid, and lethal. J Trauma Acute Care Surg_76(3):582-592

3. Spahn DR, Bouillon B, Cerny V, et al (2019) The European guideline on management of major bleeding and coagulopathy following trauma: fifth edition. Crit Care 23(1):98

4. Billeter A, Turina M, Seifert B, et al (2009) Early serum procalcitonin, interleukin-6, and 24-hour lactate clearance: useful indicators of septic infections in severely traumatized patients. World J Surg 33(3):558-566

5. Pape HC, Lefering R, Butcher N, et al (2014) The definition of polytrauma revisted: An international consensus process and proposal of the new 'Berlin definition'. J Trauma Acute Care Surg 77(5):780786

6. Pape HC, Champion HR (2015) Patient assessment in polytrauma: Current trends rely on multiple parameters to improve the prediction of complications and mortality. Injury 46(10):1875-1877

7. van Tienhoven AJ, van Beers CAJ, Siegert CEH (2019) Agreement between arterial and peripheral venous lactate levels in the ED: A systematic review. Am J Emerg Med 37(4):746-750

8. Sprengel K, Simmen H, Werner CM, et al (2016) Resuscitation with polymeric plasma substitutes is permissive for systemic inflammatory response syndrome and sepsis in multiply injured patients: a retrospective cohort study. Eur J Med. Res 21(1):39

9. Mitra B, Tullio F, Cameron PA, et al (2012) Trauma patients with the 'triad of death'. Emerg Med J 29:622-625

10. Kutcher ME, Howard BM, Sperry JL, et al (2015) Evolving beyond the vicious triad: Differential mediation of traumatic coagulopathy by injury, shock, and resuscitation. J Trauma Acute Care Surg 78(3):516-523

11. Tran A, Yates J, Lau A, et al (2018) Permissive hypotension versus conventional resuscitation strategies in adult trauma patients with hemorrhagic shock: A systematic review and meta-analysis of randomized controlled trials. J Trauma Acute Care Surg 84(5):802-808 
12. Scalea TM, DuBose J, Moore EE, et al (2012) Western Trauma Association critical decisions in trauma: management of the mangled extremity. J Trauma Acute Care Surg 72(1):86-93

13. Undurraga Perl VJ, Leroux B, Cook MR, et al (2016)_Damage-control resuscitation and emergency laparotomy: Findings from the PROPPR study. J Trauma Acute Care Surg 80(4):568-574

14. Pape HC, Rixen D, Morley J, et al (2007) Impact of the method of initial stabilization for femoral shaft fractures in patients with multiple injuries at risk for complications (borderline patients). Ann Surg 246(3):491-499

15. Horst K, Andruszkow H, Weber CD, et al Thoracic trauma now and then: A 10 year experience from 16,773 severely injured patients. PLoS One 12(10):e0186712

16. Sakellaris G, Blevrakis E, Petrakis I, Dimopoulou A, Dede O, Partalis N, Alegakis A, Seremeti C, Spanaki AM, Briassoulis G. Acute coagulopathy in children with multiple trauma: a retrospective study. J Emerg Med 47, 539-545 (2014).

17. Wang HE, Callaway CW, Peitzman AB, et al (2005) Admission hypothermia and outcome after major trauma. Crit Care Med 33:1296-1301

18. Pape HC, Giannoudis PV, Krettek C, et al (2005) Timing of fixation of major fractures in blunt polytrauma: role of conventional indicators in clinical decision making. J Orthop Trauma 19:551-562

19. van Tienhoven AJ, van Beers CAJ, Siegert CEH (2019) Agreement between arterial and peripheral venous lactate levels in the ED: A systematic review. Am J Emerg Med 37:746-750

20. Ogura T, Nakamura Y, Nakano M, et al (2014) Predicting the need for massive transfusion in trauma patients: the Traumatic Bleeding Severity Score. J Trauma Acute Care Surg 76(5):1243-1250

21. Ebadollahi S, Sun J, Gotz D, et al (2010) Predicting patient's trajectory of physiological data using temporal trends in similar patients: a system for near-term prognostics. AMIA Annu Symp Proc 2010:192-196.

22. Sankey HR (1896) The thermal efficiency of steam-engines. M.P.I.C.E 125:182-212

23. Fekete JD, van Wijk JJ, Stasko JT, et al The value of information visualization. (2008) In: Kerren A, Stasko JT, Fekete JD, North C (eds) Information Visualization. Lecture Notes in Computer Science 4950, Springer, Berlin, p. 1-18

24. Mathis B, Ma Y, Mancenido M, et al (2019) Exploring the design space of Sankey diagrams for the food-energy-water nexus. IEEE Comput Graph Appl. https://doi.org/10.1109/MCG.2019.2927556

25. Platzer A, Polzin J, Rembart K, et al (2018) BioSankey: visualization of microbial communities over time. J Integr Bioinform 15(4):20170063 
26. Ahmed MN, Toor AS, O'Neil K, et al (2017) Cognitive computing and the future of health care cognitive computing and the future of healthcare: the cognitive power of IBM Watson has the potential to transform global personalized medicine. IEEE Pulse 8(3):4-9

27. Shader RI (2016) Some reflections on IBM Watson and on women's health. Clin Ther 38(1):1-2.

28. Guo S, Lin C, Gotz D et al (2016) Understanding care plans of community acquired pneumonia based on Sankey diagram.Visual Analytics in Healthcare Workshop. https://guoshunan.github.io/files/sankey.pdf

29. Moher D, Shamseer L, Clarke M, et al (2015) Preferring reporting items for systematic review and meta-analysis protocols (PRISMA-P) 2015 statement. Syst Rev 4:1.

30. Osler T, Baker SP, Long W (1997) A modification of the injury severity score that both improves accuracy and simplifies scoring. J Trauma 43(6):922-925

31. Dübendorfer C, Billeter AT, Seifert B, et al (2013) Serial lactate and admission SOFA scores in trauma: an analysis of predictive value in 724 patients with and without traumatic brain injury. Eur J Trauma Emerg_Surg 39(1):25-34

32. Nica L, Albrecht K, Keel M, Trentz O. Independent Predictors of Early Death of Polytrauma Patients: An Analysis of 696 Patients. J Trauma Treatment 1:2-5 (2012).

33. Dienstknecht T, Rixen D, Giannoudis P, et al (2013) Do parameters used to clear noncritically injured polytrauma patients for extremity surgery predict complications? Clin Orthop Relat Res 471(9):28782884

34. Hildebrand F, Lefering R, Andruszkow H, et al (2015) Development of a scoring system based on conventional parameters to assess polytrauma patients: PolyTrauma Grading Score (PTGS). Injury 46 Suppl 4:S93-S98

35. Ahmed MN, Toor AS, O'Neil K, et al (2017) Cognitive computing and the future of health care cognitive computing and the future of healthcare: the cognitive power of IBM Watson has the potential to transform global personalized medicine. IEEE Pulse 8(3):4-9

36. Bostock M (2017) Angular Java Script Network. Updated 07.09.2018.

37. Bootstrap: Designed and built by @mdo and @ fat. Version 4.1.3. Updated 24.07.2018. https://getbootstrap.com/

38. Bostock M (2017) Data Driven Documents. Version 5.7.0. Updated 24.08.2018. https://d3js.org/

39. Brohi K, Cohen MJ, Davenport RA (2007) Acute coagulopathy of trauma: mechanism, identification and effect. Curr Opin Crit Care 13:680-685 
40. Kauvar DS, Lefering R, Wade CE (2006) Impact of hemorrhage on trauma outcome: an overview of epidemiology, clinical presentations, and therapeutic considerations. J Trauma. 60(6 Suppl):S3-S11

41. Nathens AB, Jurkovich GJ, Cummings P, et al (2000) The effect of organized systems of trauma care on motor vehicle crash mortality. JAMA 283(15):1990-1994

42. Halvachizadeh S, Pape HC (2019) Determining the patient at risk - are scoring systems helpful to develop individualized concepts for safe definitive fracture fixation and damage control techniques Injury 50,7: 1269-71?

43. Trentzsch H, Huber-Wagner S, Hildebrand F, et al (2012) Hypothermia for prediction of death in severely injured blunt trauma patients. Shock 37:131-139

44. Border JR (1995) Death from severe trauma: open fractures to multiple organ dysfunction syndrome. J Trauma 39:12-22

45. Ogura T, Nakamura Y, Nakano M, et al (2014) Predicting the need for massive transfusion in trauma patients: the Traumatic Bleeding Severity Score. J Trauma Acute Care Surg 76(5):1243-1250. 
Clinical Pathways Data Transformation (offline)
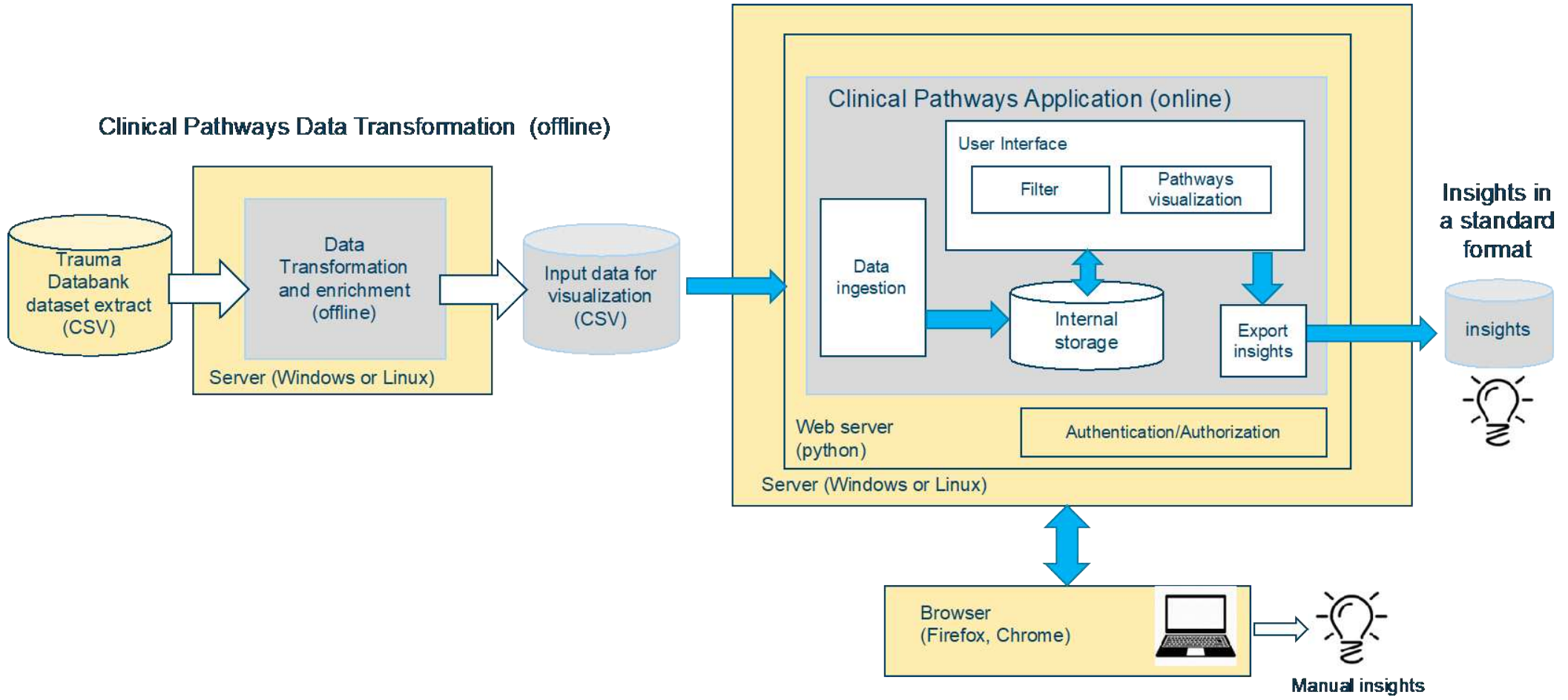

Figure 1: The digital Architecture of the Sankey visual analytics tool modified for the use in polytraumatised patients. 


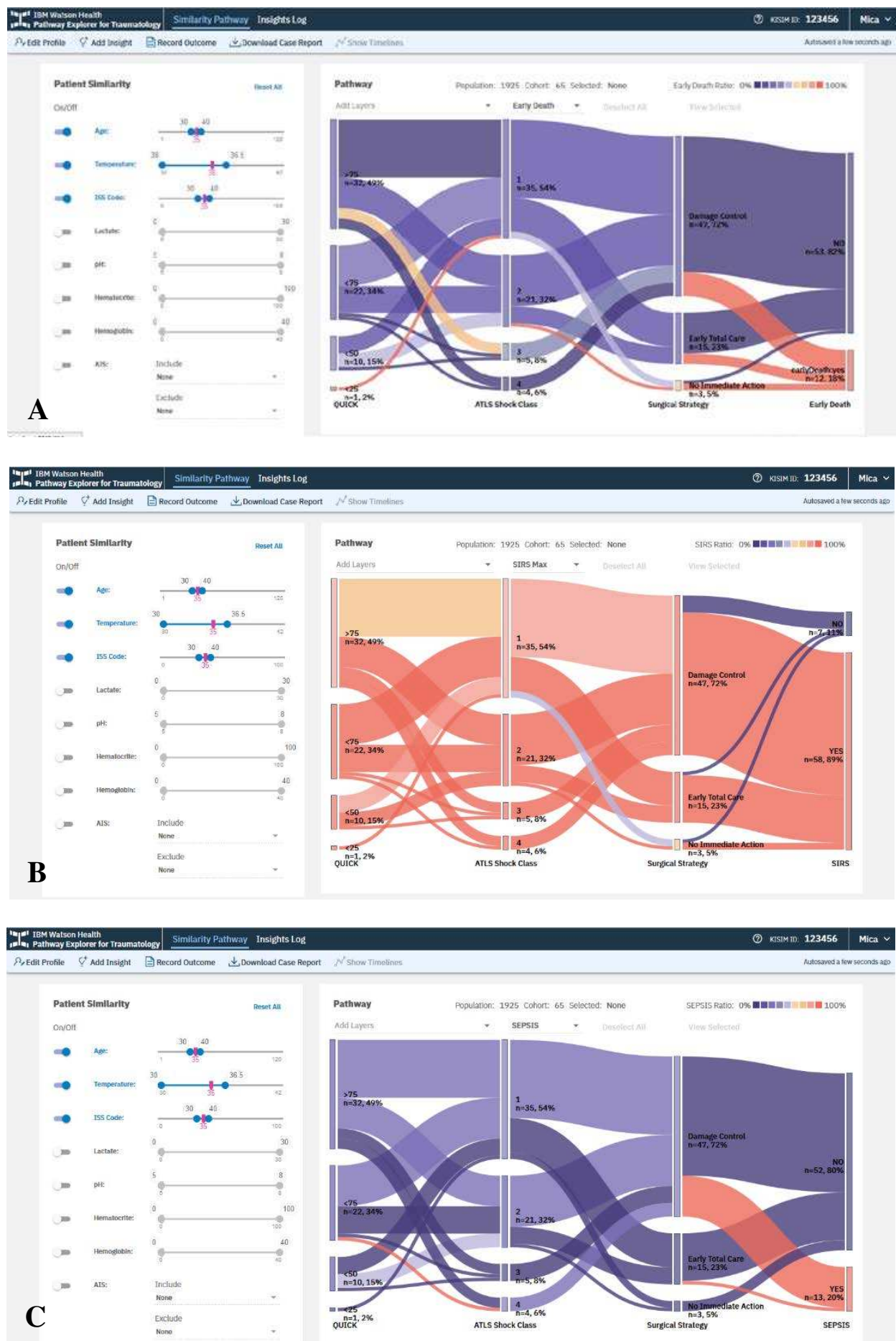
D:

Structure of the Sankey diagram and selection options for outcome analysis

\begin{tabular}{|c|c|c|c|c|c|}
\hline \multicolumn{2}{|c|}{ Patient similarity selection } & \multicolumn{3}{|c|}{ Preset Sankey visualization } & \multirow{2}{*}{$\begin{array}{c}\text { Outcome } \\
\text { Bar VI }\end{array}$} \\
\hline Bar I & Bar II & Bar III & Bar IV & Bar V & \\
\hline Age & range & \multirow{8}{*}{$\begin{array}{l}\text { Prothrombin } \\
\text { time } \\
\text { (bins: } 0-25,26- \\
\text { 50, 51-75 and } \\
\text { 75-1xx \%) }\end{array}$} & \multirow{8}{*}{$\begin{array}{c}\text { ATLS shock } \\
\text { I-IV }\end{array}$} & \multirow{8}{*}{$\begin{array}{c}\text { Surgical } \\
\text { strategy (bins: } \\
\text { early total care, } \\
\text { damage } \\
\text { control, none) }\end{array}$} & \multirow{8}{*}{$\begin{array}{c}\text { SIRS or sepsis } \\
\text { or Death (bins: } \\
\text { yes or no) }\end{array}$} \\
\hline Temperature & range & & & & \\
\hline ISS & range & & & & \\
\hline Lactate & range & & & & \\
\hline $\mathrm{pH}$ value & range & & & & \\
\hline Hematocrit & range & & & & \\
\hline Hemoglobin & range & & & & \\
\hline AIS & range & & & & \\
\hline
\end{tabular}

Figure 2: Sankey diagrams of the analysis of Pathway Explorer for Traumatology of a hypothetical patient with an age of 35 years, an ISS of 35 and a temperature ad admission of $35^{\circ} \mathrm{C}$. This screenshot extractions (A-C) show the pathways to the three outcomes: early death (A), SIRS (B) and sepsis (C). The percentage of the patient cohort suffering the corresponding outcome is given as a colored code at the upper right of each screen shot (A-C). The X-axis does not represent a timeline but an interchangeable event sequence. The given events are binned and the patients are assorted according the fulfilling of these events. Each of the binned events can be chosen and the conditions for the chosen outcome can be displayed. The thickness of the bars indicates the cohort size and the color code indicated the percentage of the cohort to develop an adverse outcome (SIRS, sepsis and early death). Figure 2D shows the detailed bardescription of the Sankey output. All layers are electable for each bin, as well as the respective cohort. Quick: Prothrombin Time. 


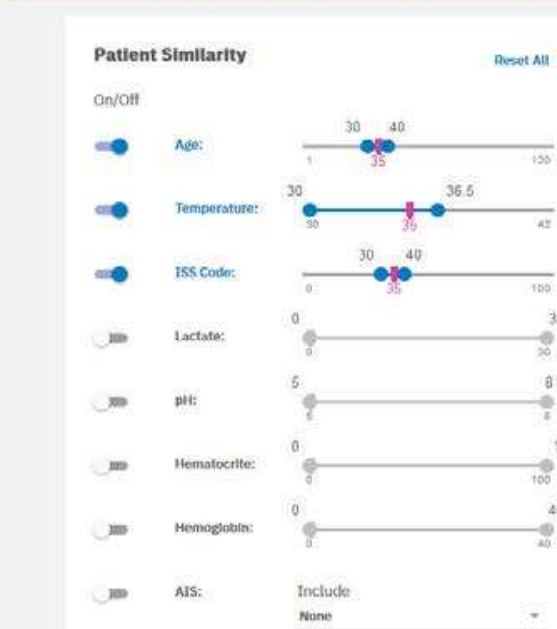

A
Pathway

Add Layery

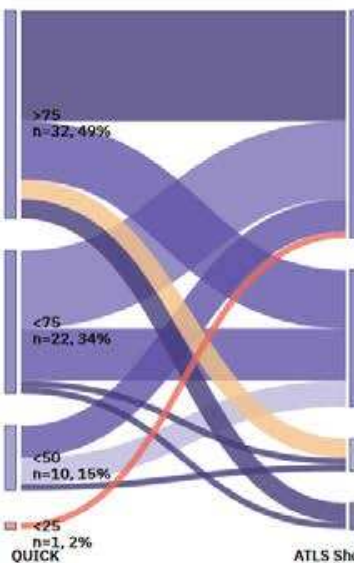

Ropulation: 1925 cotort 65 selected None

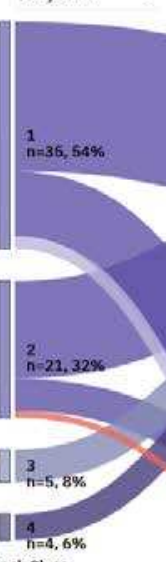

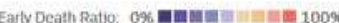
Demolect ail

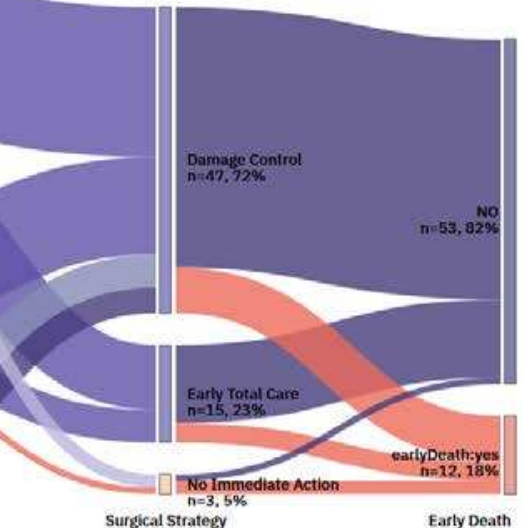

Autosaved a tew seconds ase

2, Edit Profile Add Insight $\quad$ Record Outcome $\downarrow$ Download Case Report $N$ show Timelines

\section{Pathway}

Population: 1925 Cohort: 65 Selected None

on/oft

$\rightarrow$ AgE:

- Temperature:

- 155 code:

- Lactate:

m ph

mis Hematocrite

Hemoglobin:

$=$ NS

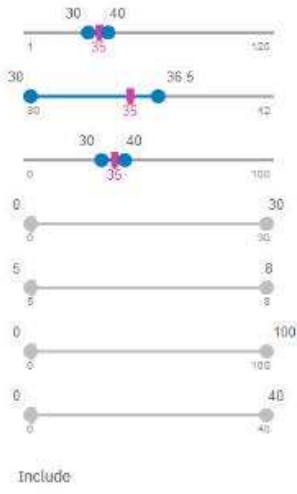

Add Layers

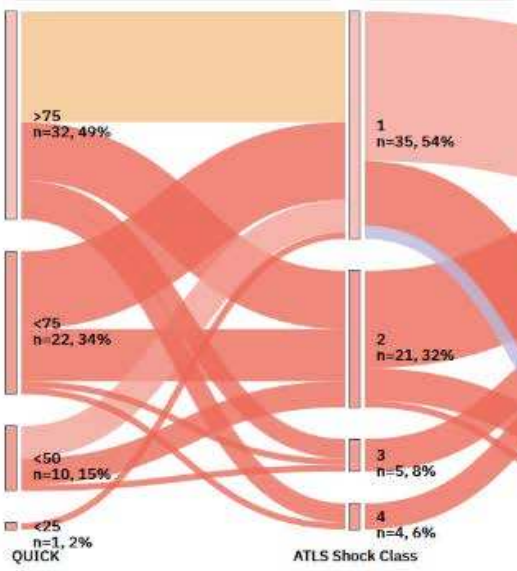

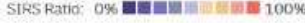

nine seltecied

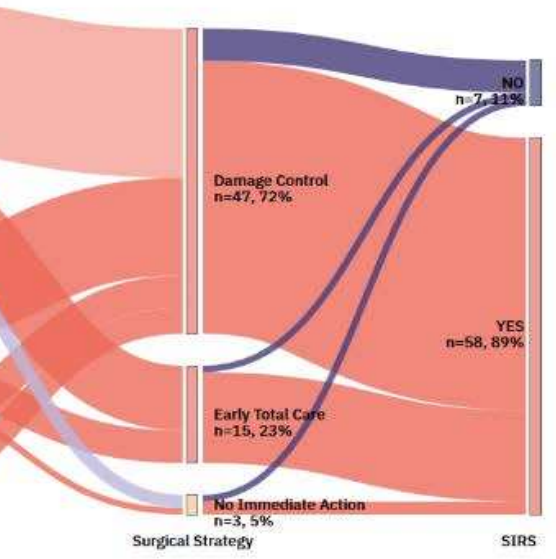

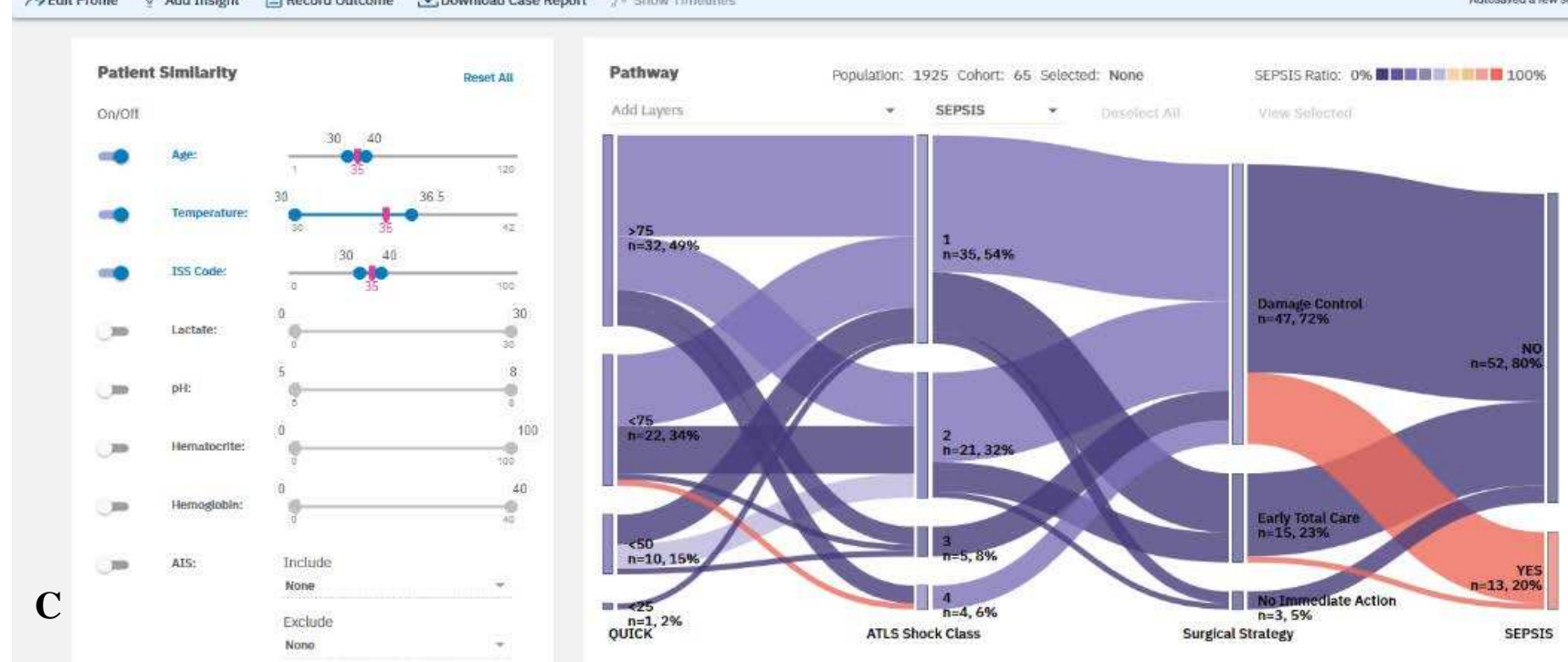




\begin{tabular}{|c|c|c|c|c|c|}
\hline \multicolumn{2}{|c|}{ Patient similarity selection } & \multicolumn{3}{|c|}{ Preset Sankey visualization } & \multirow{2}{*}{$\begin{array}{c}\text { Outcome } \\
\text { Bar VI }\end{array}$} \\
\hline Bar I & Bar II & Bar III & Bar IV & Bar V & \\
\hline Age & range & \multirow{8}{*}{$\begin{array}{l}\text { Prothrombin time } \\
\text { (bins: } 0-25,26-50 \text {, } \\
51-75 \text { and } 75-1 \mathrm{xx} \\
\% \text { ) }\end{array}$} & \multirow{8}{*}{$\begin{array}{c}\text { ATLS shock I- } \\
\text { IV }\end{array}$} & \multirow{8}{*}{$\begin{array}{l}\text { Surgical strategy } \\
\text { (bins: early total } \\
\text { care, damage } \\
\text { control, none) }\end{array}$} & \multirow{8}{*}{$\begin{array}{l}\text { SIRS or sepsis or } \\
\text { Death (bins: yes } \\
\text { or no) }\end{array}$} \\
\hline Temperature & range & & & & \\
\hline ISS & range & & & & \\
\hline Lactate & range & & & & \\
\hline $\mathrm{pH}$ value & range & & & & \\
\hline Hematocrit & range & & & & \\
\hline Hemoglobin & range & & & & \\
\hline AIS & range & & & & \\
\hline
\end{tabular}


Table 1: Time table for development and implementation of the Watson Trauma Health application

Pre - meeting scientific sessions

-, Kick-off session: $27^{\text {th }}$ Intl. Course Polytrauma Management

- in person discussions and preparation for funding application

- Follow-up $28^{\text {th }}$ Intl. Course on Polytrauma Management

Munich GER March 2017

Zurich, SUI April/May 2017

Bucharest, ROM May 2017

Application for funding

- in person meeting (USZ) to discuss grant application

Zurich, SUI April/May 2017

- preparatory meetings (IBM and USZ) (distribution of funding) Zurich, SUI May, 2017

- Application according to deadline Research fund USZ

Zurich, SUI June 30, 2017

- Approval of funding (200.000 SFR)

Zurich, SUI August 2017

Preparatory meetings (USZ: LM, HCP)

Zurich, SUI July/Aug 2017

- Review of published data

- Review of existing data base parameters

- Selection of parameters for risk profile and individual data (USZ)

- Implementation into Watson Health Sankey diagram (IBM)

Preliminary discussion

$29^{\text {th }}$ International Polytrauma course, DKOU

Berlin, GER Oct 2017

Invitations and information for users

Zurich, November 2018

- Pre-circulation of definitive time line

- Pre-circulation of topics

- Pre-circulation of background materials

- preparative meeting for data implementation development

(USZ; LM and HCP) and process (IBM; CN, PB, AY)

Zurich Consensus conference, January 2018 (USZ and IBM)

Presentation: of Sankey Watson Trauma Health

Zurich, January 2018

Follow up of the 'Sankey Working Group "Polytrauma”, Valencia, ESP May, 2018

Consensus discussion at the $\mathbf{2 3}^{\text {th }}$ Int. Polytrauma Course, Z Zurich Sept., 2018

Discussion of further proceedings during 2018 /19

Use/Assessment of Watson Heath Trauma Sankey tool in Z Zurich, June-2019-2019

Presentation and discussion in Polytrauma section meeting Prague, ESTES 2019

Grand round presentations (Allegheny General Hospital) Pittsburgh, USA, June 2019

Grand round presentation (Indi Health System) Indianapolis, USA, June19 
Table 2: Structural differences of primary versus secondary selection data, and treatment induced data that determine the subcohort outcomes

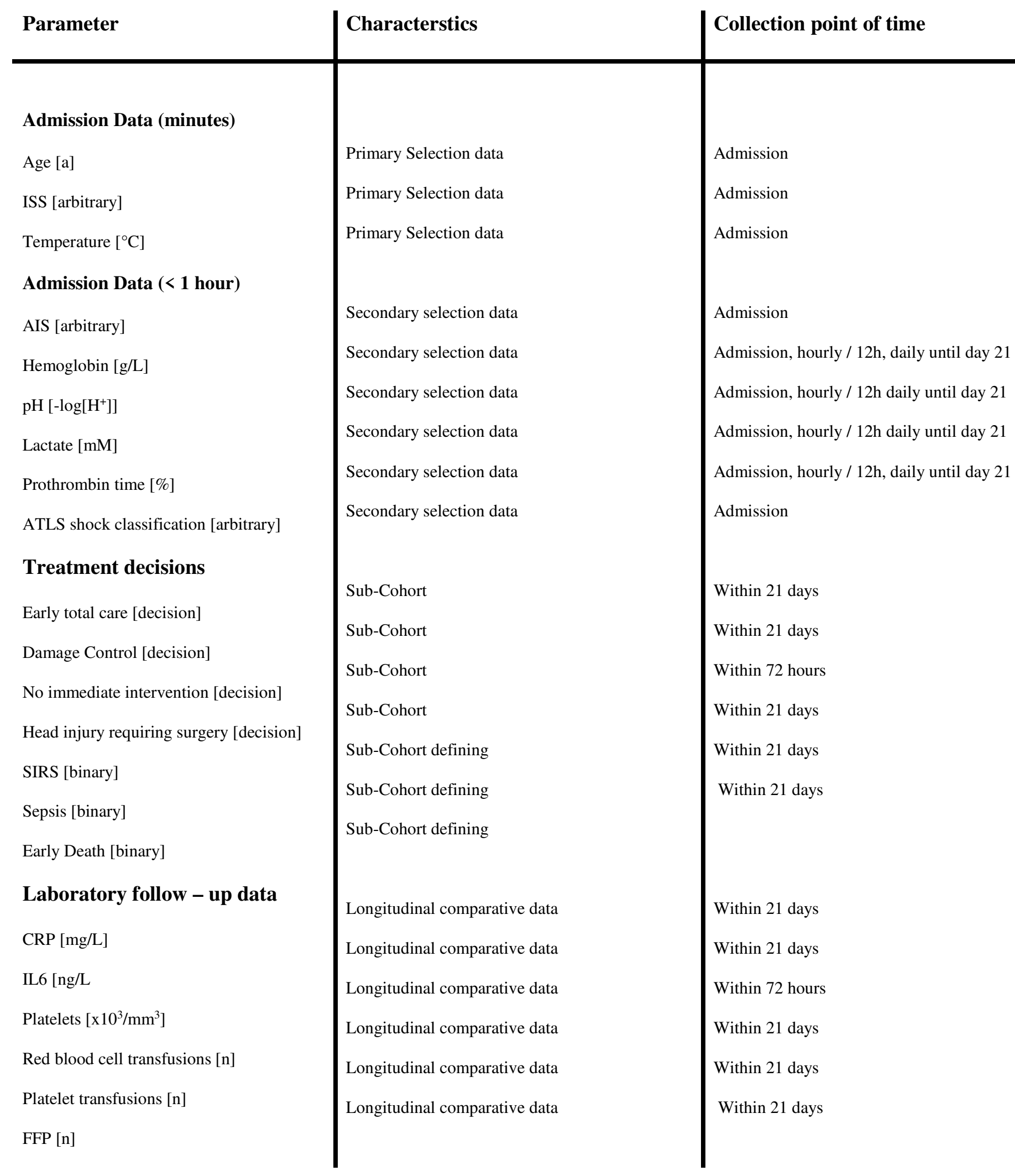

